Running head: Bariatric surgery and long term psychosocial quality of life

\title{
The effectiveness of bariatric surgery on long term psychosocial quality of life - a systematic review
}

Sandra Jumbe ${ }^{1}$, Claire Bartlett ${ }^{2}$, Samantha $L$ Jumbe ${ }^{3}$ and Jane Meyrick $^{4}$

${ }^{1}$ Health and Care Research Wales Workforce, Cardiff, UK ${ }^{2}$ School of Optometry and Vision Sciences, Cardiff University, UK ${ }^{3}$ Faculty of Health and Life Sciences, University of the West of England, Bristol, UK ${ }^{4}$ Department of Psychology, Faculty of Health and Life Sciences, University of the West of England, Bristol, UK

Word count (exc. figures/tables): 5250

${ }^{*}$ Requests for reprints should be addressed to: Sandra Jumbe, Health and Care Research Wales Workforce, South East Wales Research Network, 12 Cathedral Road, Cardiff CF11 9LJ (email:Sandra.Jumbe@live.uwe.ac.uk) 
Running Title: Bariatric Surgery and long term Psychosocial Quality of Life

\section{ABSTRACT}

Background: Little is known about the psychological effects of bariatric surgery. This review aimed to assess long term effectiveness of bariatric surgery on psychosocial quality of life (QoL) of obese adults compared to non-surgical interventions.

Methods: A systematic review of the literature was conducted, six electronic databases, plus other relevant sources were searched from inception to January 2014. The main outcome sought was a QoL measure with a minimum 12 months follow-up. Three reviewers screened records, extracted data and independently read through full articles for eligibility and quality using standardised forms. Findings were analysed using narrative synthesis.

Results: Of 4383 identified references included in the review, 11 studies showed bariatric surgery to be effective long term for overall QoL than non-surgical treatments with specifically modest benefits psychosocially. Significant improvements in psychosocial QoL alongside improved physical QoL were observed after 2 year follow-up post-surgery compared to nonsurgical interventions. However improvements in psychosocial QoL after surgery at 10 year follow up were minimal when compared to non-surgical interventions. When compared to untreated control groups, long term psychosocial QoL did not improve after bariatric surgery despite major improvements in physical QoL, significant weight loss and co-morbidities.

Conclusions: Results on long term psychosocial QoL remain uncertain with some suggestion of psychological disorder persisting. This highlights need for psychological intervention postsurgery and further research to provide more data on long-term psychosocial QoL following bariatric surgery.

Key words: bariatric surgery, weight loss, psychosocial, quality of life 
Running Title: Bariatric Surgery and long term Psychosocial Quality of Life

\section{BACKGROUND}

Obesity is a major health problem worldwide and has reached epidemic proportions in Western society and the UK [1] [2], making it an extremely important public health issue in its contribution to major diseases. [3-5]. Obesity can also be a psychosocial burden, often resulting in social stigma and negatively affecting individuals' underlying mental health and self-esteem [6-8].

Obesity can be managed using dietary advice, exercise, lifestyle changes and medication to induce weight loss. Weight loss of 5 to $10 \%$ has been associated with significant reductions in co-morbidities and mortality [9]. However it is very difficult for obese adults to lose weight through lifestyle choices in these circumstances and a high proportion who attempt to lose weight through traditional weight loss methods either experience minimal weight loss maintenance or regain the weight over time [10]. Bariatric surgery (BS) is increasingly used when all other treatments such as physical activity and diet have failed [11,12] with statistics from the Health \& Social Care Information Centre showing a 6 fold increase in the procedure due to obesity diagnosis between years $2002-03$ to $2012-13$ in the UK $[13,14]$.

Current literature has shown BS to be a more effective treatment for severe obesity as part of an overall weight management strategy, when compared to the traditional behavioural interventions [15], achieving significant weight loss in people with a BMI of $30 \mathrm{~kg} / \mathrm{m}^{2}$ (including those with more severe obesity), longer term maintenance of weight loss and improvements in co-morbidities compared to non-surgical obesity management. Aside from significant long term weight loss, long-term studies such as Robinson [16] and systematic reviews have demonstrated recovery from diabetes, improvement in cardiovascular risk factors, and a reduction in mortality following BS [17-19]. This approach to obesity takes a clear physiological perspective to health improvement which may mask underlying psychological issues. 
A growing awareness of the importance of psychological and social influences on health and illness has led to the development of a biopsychosocial framework [20] for research and intervention, influencing the inclusion of assessing patients' health-related quality of life (HRQoL) in clinical and research settings, where the inseparable nature of physical, psychological and social factors are taken into account [21]. Saying this, research into psychosocial QoL in bariatric surgery patients is sparse. This may be because data collected in this area is mainly based on subjective self-reported HRQoL measures which tend to compare unfavourably to 'stronger' evidence from physiological, psychological and biochemical tests [21-26]. Another reason may be the fact that health professionals working in this area are largely trained in the medical model $[27,28]$ where focusing on physiological issues is more natural than adopting the biopsychosocial approach to patient care.

However, a substantial proportion of people eligible for BS have a history of eating disorders or mental health problems $[29,30]$. Research looking at psychological predictors of obesity presents complex findings and is not well understood [31-33]. However, some studies have found certain psychological factors in obese individuals like depressive symptoms, post-surgery emotional struggles and dichotomous thinking that may contribute to weight regain [34-36] Can weight loss through BS alone address these underlying psychological conditions that possibly contributed to the obesity in the first place?

The National Institute for Health and Clinical Excellence (NICE) commissioning guidance for bariatric surgical service [37] highlights the need for a multidisciplinary approach which includes psychological intervention before and after surgery in order to achieve high quality obesity treatment for patients. However despite these recommendations and recognition that this patient group experiences long-term psychological and social difficulties, the provision of 
Running Title: Bariatric Surgery and long term Psychosocial Quality of Life

psychological interventions alongside and following BS is not well established in the UK, with focus mainly being on physical aspects like diet and exercise in the NHS [38].

\section{Why is it important to do this review?}

Previous systematic reviews reporting on quality of life (QoL) as a main or secondary outcome, concluded that although BS improves short term (two years) health-related quality of life (HRQoL) in obese adults, longer term (ten years) effects were less clear [39] with some measures showing significantly greater change after surgery, but not others. However, not all the studies included in the systematic reviews assessed QoL and outcome data for QoL was unclear for almost half of the studies reviewed, thus making it difficult to make clear judgements about the impact of weight loss interventions on longer term quality of life [18 \& 19]. As such both reviews highlighted a need for good-quality, long-term randomised controlled trials (RCTs) that include an assessment of patient QoL following BS.

Assessing psychosocial QoL within BS could contribute to greater understanding of the postsurgical psychological and behavioural changes in patients. BS specialists and service providers highlight the importance of the patient adjusting their behaviour accordingly to accommodate compliance to a strict diet and exercise post-surgery. Achieving significant weight loss even through BS is a journey of behavioural adjustment over years and the link between psychological distress and obesity may continue on after BS [40,41]. It is therefore important to establish whether psychosocial QoL improvements following surgery are stable and prolonged enough to buffer long term behavioural change and help reduce the psychological distress.

This review aims to find out whether BS is more effective on long term QoL compared to nonsurgical behavioural interventions that try to tackle the underlying psychosocial factors 
associated with obesity. More specifically, does BS alone improve psychosocial QoL in individuals in the long term?

\section{METHODS}

\section{Types of studies}

RCTs, controlled clinical trials and prospective cohort studies comparing surgical interventions with non-surgical treatment (behavioural OR medical management OR no treatment) reporting on all the following:

- Original research on pre and postoperative QoL or psychosocial wellbeing variables following BS.

- Postoperative QoL measures for a minimum of 12 months. No maximum cap on follow up period was imposed. No discreet definition of long term was presented however, following previous review findings [42] studies reporting over 5 year follow up periods were classed as longer term.

\section{Participants}

Adults defined as aged 18 years and over, fulfilling standard definition of obese, i.e. BMI of 30 or over according to relevant national guidelines. Studies including participants with a previous history of BS were excluded.

\section{Types of interventions}


Running Title: Bariatric Surgery and long term Psychosocial Quality of Life

Any type of currently performed bariatric surgery procedure compared to non-surgical treatment. Non-surgical treatments included usual obesity management or usual care, behavioural/medical management inclusive of low calorie diet, physical exercise, psychological therapies or counselling and medication. Studies assessing QoL outcomes between types of bariatric surgery only were excluded.

\section{Types of outcome measures}

- Quality of life (QoL), measured using a validated instrument

- Psychological wellbeing, measured using a validated instrument

\section{Search methods for identification of studies}

\section{Electronic searches}

The following electronic databases were searched for identifying potentially eligible studies:

- The Cochrane Library (07/11/2015);

- PubMed via NCBI (until 07/11/2015);

- MEDLINE (until date 07/11/2015);

- PsychINFO via EBSCO (until 07/11/2015);

- ScienceDirect (until 07/11/2015);

- JSTOR (until 07/11/2015).

Studies were identified from these databases by searching all years until the end dates specified above.

\section{Searching other resources}


Running Title: Bariatric Surgery and long term Psychosocial Quality of Life

Key journals' tables of contents (International Journal of Obesity, The European Journal of Obesity, Obesity Reviews and Obesity Surgery) were hand searched, and reference lists of relevant trials and systematic reviews identified were examined, up to 7th November 2015. Several authors were also contacted to obtain additional information for five unpublished trials, four of which were listed as completed. The fifth trial recently finished recruitment and is in follow-up [43]. Only one of the authors [44] from the four completed unpublished trials responded, stating the trial was in the process of data analysis.

\section{Data collection and analysis}

\section{Selection of studies}

One reviewer independently screened through all the titles and citations retrieved from the database searches. Eligibility criteria were applied to the full article using a standardised form by two reviewers independently. Studies not available in English language were excluded due to lack of funding for translating services.

\section{Data Extraction and Analysis}

Data on participants, interventions, outcomes and quality of the studies was extracted from all included papers and tabled. Specifically, information on changes in QoL from baseline (before surgery) to post surgery and weight change (e.g. BMI, percent excess weight change) was collected if reported. Study characteristics and outcome data were extracted by one reviewer (SJ) and checked independently by another (CN or SLJ).

Study quality was assessed using the Effective Public Health Practice Project (EPHPP) "Quality Assessment Tool for Quantitative Studies", developed for use in public health research [45] a robust tool with appropriate psychometric properties to adequately assess quality of evidence 
Running Title: Bariatric Surgery and long term Psychosocial Quality of Life

[46]. Included studies were assessed for homogeneity and synthesis via meta-analysis ruled out due to the extreme heterogeneity in participant selection criteria, interventions e.g. two different BS procedures within a surgery group) and QoL outcome measures therefore pooling results according to any of these characteristics would leave highly heterogeneous groups. Moreover, a meta-analysis could not be done because of variations in study design and lack of RCTs amongst the included papers. Indeed not all healthcare questions can be addressed by RCTs, and meta-analysis in systematic reviews is not always possible or sensible $[47,48]$. Ignoring issues of heterogeneity and assuming homogeneity in order to justify meta-analysis often results in misleading findings [49]. Instead a narrative analysis was conducted as a best case alternative, able to cope with this variation [50] using methodological guidance outlined in Thomas et al [45] and reported according to the framework recommended by the Preferred Reporting Items for Systematic Reviews and Meta-Analyses (PRISMA) statement (www.prismastatement.org), providing an analysis of the relationships within and between studies and an overall assessment of the robustness of the evidence.

\section{RESULTS}

Firstly an overview of the included studies is provided including study quality and participant characteristics, followed by analyses of QoL outcomes following BS compared to non-surgical treatments with specific focus on psychosocial QoL components.

Out of the 4383 references yielded from the electronic databases search and 49 references from other sources, de-duplication and removal of irrelevant titles provided 1443 references. From subsequent screening, selection against set criteria and independent reviewing of 127 full papers (see Supplementary Material 1) by three authors [SJ, CN and SLJ] for inclusion and quality, 11 studies fulfilled the inclusion criteria (Figure 1). These studies compared contemporary bariatric procedures (Gastric Bypass, Gastric Banding and Banded Gastroplasty) 
Running Title: Bariatric Surgery and long term Psychosocial Quality of Life

to several non-surgical weight loss treatments, obese treatment seeking groups or obese control groups. Table 1 shows a descriptive summary of all included studies.

\section{Quality}

Two of the eleven included studies were RCTs [51,52]. Six of the studies included in this review had a strong overall quality rating, according to the EPHPP quality assessment tool used (Table 2). The other five were rated as moderate. Drop-out rates for each group and reasons for dropout were reported by all of the studies apart from Batsis et al [53], as study data was collected using a onetime retrospective survey. The strength of scientific quality for each individual study is covered alongside findings in the narrative synthesis.

\section{Representativeness}

All participants from included studies were severely obesity, with a common definition of BMI being greater than 40 or the additional criteria of BMI equal or greater than 30 with co-morbid disease. One participant in Buddeberg-Fischer et al [54] with a BMI of $31.6 \mathrm{~kg} / \mathrm{m}^{2}$ had special indications for BS. The most commonly cited co-morbidities were diabetes and cardiovascular disease. The individual study sample size ranged from 43 to $1276[52,39]$. The majority of participants in the studies were female and mean age ranged from 39 years [55] to 48 years [39] which is representative of the BS population in the UK [14]

Group differences in baseline characteristics were noted in most of the studies apart from the four controlled trials [51, 52, 56, 39] and Buddeberg-Fischer et al [54]. The general trend being that the surgery group tended to be significantly younger, with greater weight or BMI than the no surgery group [55] or population based obese control group [57-60]. Batsis et al [53] reported more males, lower incidence of diabetes and lower BMI in their no surgery group compared to 
Running Title: Bariatric Surgery and long term Psychosocial Quality of Life

the surgery group. Buddeberg-Fischer et al [54] also found within group differences in their surgery group where all patients with BMl equal or greater than 50 received Roux-en-Y gastric bypass (RYGBP). The rest of the surgery group received laparoscopic adjustable gastric banding (LAGB).

\section{Outcomes measures}

Measures of weight change reported by studies ranged from BMI, change in BMI, weight, weight loss, percent weight loss and percent excess weight loss. All the studies reported measures of variability such as confidence intervals or standard deviations. However, the direct comparison of values between studies is questionable due to the different measures reported.

Similarly QoL was assessed using various measures, most commonly the SF-36 Health questionnaire and Impact of Weight on Quality of Life-lite (IWQoL-lite) questionnaire. Other measures used were the EuroQol 5 Dimensions (EQ-5D) scale [51, 52], Linear Analogue Self Assessment (LASA) questionnaire and SF-12, a short form of the SF-36 [53], the Psychosocial Stress and Symptom Questionnaire [54], the Current Health Scale, Mood Adjective Checklist (MACL), Obesity-related Problems Scale (OP) and Sickness Impact Profile (SIP).

\section{Long term follow up}

Duration of QoL follow-up ranged from 12 months [51, 52, 55] to 10 years [39] however, most studies followed up participants between 2 to 6 years (Table 1). To assess long term effectiveness of BS on psychosocial QoL, QoL outcomes between BS and non-surgical interventions were compared over time. Two subgroups were identified in the non-surgical group, namely; those receiving lifestyle modification or conventional treatment and population controls who received no treatment. Therefore analysis was split further into BS vs. non-surgical 
Running Title: Bariatric Surgery and long term Psychosocial Quality of Life

weight loss treatments and BS vs. controls. As described in Table 1, the included studies predominantly used (laparoscopic) gastric banding and gastric bypass.

\section{Quality of Life in Bariatric Surgery vs. Non-surgical Weight Loss Treatments}

Four studies were identified in this group, namely two RCTs [51, 52], a prospective cohort study [55] and one controlled trial [39], reporting QoL outcomes over a 1 year follow up period [51, 52,

55] and at 10 years [39]. Canetti et al [55] examined psychosocial factors on the treatment outcomes of BS and a weight-loss program (which combined diet, behaviour modification and exercise). The RCTs assessed QoL outcomes in obese Type 2 Diabetic patients following LAGB [51] or RYGBP [52] compared to an intensive diabetes medical weight management program (WhyWAIT). QoL was measured using the SF-36 health questionnaire in all three studies although the RCTs also used the EQ-5D and IWQOL. At 1 year follow up, all three studies found that both the BS and non-surgical treatment groups experienced QoL improvements over time and evidenced more significant weight loss in the BS groups. Specific to SF-36 scores, the BS patients in Canetti et al [55] showed significantly greater improvement in every QoL subscale compared to the diet group. The diet group showed significant improvements in three of the eight SF-36 subscales (subscales unspecified) and the total score over the year. In Halperin et al [52] QoL improvements based on SF-36 scores and EQ-5D visual analogue scores were similar between groups. However a significant change in mental health scores from baseline to one year was noted within the WhyWAIT group. The paper also states no significant change within or between groups for EQ-5D index scores (figures unreported). Again with the IWQOL, QoL scores improved significantly following both RYGB and Why WAIT after a year, however the magnitude of improvement was significantly greater in the BS group. The other RCT [51] also found QoL scores from baseline to one year across all measures improved similarly between the two groups. However, a significant within group QoL improvement in total and mental health scores of the SF-36 scores for the WhyWAIT group was observed after one year. 
Running Title: Bariatric Surgery and long term Psychosocial Quality of Life

Overall, these studies show moderate quality evidence of similar improvements in QoL from BS and non-surgical weight loss treatments in obese individuals with some inference of more positive impact on psychosocial QoL following medical weight management and more positive impact on physical QoL after BS at one year follow up.

Results from Karlsson et al [39] who assessed health related QoL in 1276 participants using a battery of generic and obesity specific measure over ten years, showed significant improvements in all health related QoL measures in BS patients compared to patients receiving conventional treatment (described as unstandardised and varied treatment regimens according to local practices) overall. In the BS group, QoL improvements peaked during the first year of weight loss, whereas the weight regain phase (mainly between 1 and 6 year follow-up) was accompanied by a gradual decline in health related QoL. The latter period of 6 to 10 years saw relatively stable observations in both weight and QoL. Overall at 10 years increases in all health related QoL domains in the BS group were noted compared to baseline. The conventional group observed small initial improvements which were mostly lost within 2 years with some health related QoL domains like obesity-related psychosocial problems and anxiety improving at the end of the observation period. However long-term outcomes were mixed for the conventional group. This study provides strong evidence that BS has a significantly positive long term effect on health related QoL (including psychosocial functioning) when compared to conventional weight loss treatment.

\section{Quality of life in Bariatric Surgery vs. Control Groups}

Studies in this group comprised one controlled trial and six prospective longitudinal cohort studies reporting QoL results over a follow up period from 2 to 6 years. There were two studies that assessed QoL in BS participants against controls for two years [57, 58]. Both studies along with Koloktin et al [60] and Adams et al [59] form part of the Utah Obesity Study, an ongoing 
prospective study of GB patients that includes 2 obese control groups; those seeking but not undergoing GB (primarily due to insurance cover restrictions) and a population based obese group not seeking BS. Both studies [57 \& 58] reported significantly greater improvements in overall QoL in the GB group than the controls with moderate to very large effect sizes for the GB group in psychosocial QoL compared to small to moderate effects sizes for the other groups [57]. However, the group difference in mean QoL scores between BS and control cohorts was less significant in the SF-36 mental component $(p<0.01)$ compared to the physical component and overall QoL $(p<0.0001)$ [58]. Overall, both studies show strong quality evidence that BS significantly improves psychosocial QoL when compared to no treatment.

A prospective trial by Nickel et al [56] looking at QoL after gastric banding found generally reduced QoL and health state for people with a high BMI at baseline regardless of which group they were in. At 3 years post-surgery, the gastric banding patients showed significantly greater improvement in all eight SF-36 scales than the no surgery group. Moreover, a reduction in BMI showed a prolonged positive effect on both psychological and physical symptoms. The lack of group differences in baseline characteristics and the high follow up rate of $88 \%$ of this trial provides strong quality evidence of a significantly positive effect on psychosocial QoL following BS when compared to no treatment.

Two studies assessed QoL following BS up until four years. Batsis et al [53] examined the relationship between QoL and psychological status following RYGBP using the LASA and SF12. The LASA QoL score improved from baseline to follow up after 4 years by $138 \%$ (surgery) and $31 \%$ (no surgery), a significant percentage difference between the groups. The surgery group also had considerable symptomatic improvement and higher self-reported exercise tolerance at follow up. Reported SF-12 scores at 4 years also showed significant improvement in overall QoL in the surgery group compared to controls. However it might be worth noting that the 
Running Title: Bariatric Surgery and long term Psychosocial Quality of Life

LASA was the only QoL measure taken at baseline and follow up. Considering the fact that it only asks one global QoL question, one can question the tool's ability to specifically assess psychosocial QoL. Interestingly, the study reports that when comparing SF-12 scores in all patients between the follow up periods of $<2$ years and $>2$ years, no significant difference in mental component score was found between the BS and no surgery groups. However, the study reported a positive correlation between the two scales and overall, it provides moderate quality evidence that BS significantly improves general QoL compared to no treatment but not psychosocial QoL necessarily.

Buddeberg-Fischer et al [54] investigated weight loss, psychosocial stress and psychosomatic symptoms between patients undergoing and not undergoing BS over a four and a half year period. 69 out of 131 participants from two specialised surgery units chose to have LAGB or RYGBP. All patients were followed up at two years, and four and a half years via telephone interviews. At 4 years, all patients seemed to rate their psychosocial wellbeing as 'good' regardless of whether they had undergone surgery or not. This is despite a significant reduction in BMI in the surgery group compared to the no surgery group. Both groups also showed a significant improvement in depressive symptoms. This study provides moderate quality evidence of no significant difference in long term psychosocial QoL following BS when compared to no treatment.

In the longer term Utah Obesity Group studies $[59,60]$ there was an absence of significant improvement in the SF-36 mental component score for the GB group after six years, contrary to marked significant improvements in the physical component scores and overall QoL score [59]. Significant improvements were evident in all the physical domains of QoL measures but only some of the psychosocial domains significantly improved after 6 years in the GB group compared with the controls [60]. QoL scores remained stable from 2 to 6 years, where small 
Running Title: Bariatric Surgery and long term Psychosocial Quality of Life

decreases in QoL were linked to some weight gain. Overall, both studies show strong quality evidence that BS does not significantly improve long term psychosocial QoL when compared to no treatment.

Overall four out of seven studies comparing BS to control groups found good evidence that long term psychosocial QoL does not improve following BS compared to no treatment at all. Figure 2 outlines the post-surgery trajectory of psychosocial QoL from the studies using the SF-36 and SF-12 between BS and control groups over time. Five of the seven studies had an overall strong quality rating.

\section{Quality of Life Outcomes in relation to Type of Procedure among Included Papers}

An attempt was made to look at the relationship between type of surgical procedure (gastroplasty, gastric banding and gastric bypass) and reported QoL outcomes (Table 3). Three studies [39, 54 \& 55] used two types of BS within their surgery group according to the surgeon's discretion or patient's choice. None of these studies reported or made QoL comparisons in relation to type of surgical procedure. However studies have shown gastric bypass to be associated with better quality of life outcomes compared to gastric banding and gastroplasty [6163]. Data available for both within study comparisons and across study comparisons showed more percentage excess weight loss (\%EWL) for gastric bypass participants compared to the other two procedures. This again is in line with previous statistical findings in weight loss surgery literature $[18,62,64-65]$. Weight loss tended to slow down with longer follow up and in some cases weight regain occurred [59]. No percentage weight loss (\%WL) or \%EWL was reported in Nickel et al [56].

\section{Quality of Life Outcomes in relation to Gender among Included Papers} An attempt was made to look at the relationship between gender of participants and QoL 
Running Title: Bariatric Surgery and long term Psychosocial Quality of Life

outcomes following BS (Table 3). None of the studies included in this review conducted such analyses. Batsis et al [53] who conducted a regression analysis looking at predictors of increased QoL amongst their gastric bypass group suggested gender was not a significant predictor.

\section{DISCUSSION}

General findings from four studies of moderate to strong scientific quality show that psychosocial QoL is initially better within the first year of follow up in non-surgical intervention cohort. However this trend is not sustained after ten years, with the BS cohort showing significantly better overall QoL outcomes compared to non-surgical interventions. However in the 10 year follow up study [39] the degree of improvement in psychosocial QoL in the surgery group was minimal after 6 years despite the significant improvement in physical QoL, weight loss and co-morbidities, bringing into question the ability of BS to impact mental and social issues in this patient group and raising the need to improve theoretical pathways around psychology issues, obesity, cause and effect. When compared to no intervention, long term psychosocial QoL does not appear to improve following BS, despite significant improvements in physical QoL over time. Overall these results demonstrate moderately strong evidence to suggest that BS is not more effective at improving psychosocial QoL in the long term when compared to obese control groups, even when coupled with positive outcomes like significant weight loss and improvements in comorbidities like diabetes and cardiovascular disease. Therefore, it seems inducing significant weight loss alone by means of BS may not address longer term psychological issues like depression, body dissatisfaction and problematic eating behaviours $[66,67]$ that may be initially present in the individual suffering from obesity. 
Running Title: Bariatric Surgery and long term Psychosocial Quality of Life

This review demonstrates how persistent psychological factors linked to obesity can be and reinforces recommendations from the NICE commissioning guidance to provide psychological support after BS [11]. More crucially it shows that there is no clear study comparing the health outcomes of psychological treatment and physical treatment (BS) of obesity. Overall more long term studies in this area are needed that focus on psychosocial QoL and wellbeing of individuals following BS and a greater potentially useful adoption of theory building across life course approach around obesity.

The variety of measures used amongst the included studies means validity of QoL ratings comparisons are difficult to ascertain. The measures ranged from 'generic' scales, which are used and validated across a broad range of chronic diseases, to 'specific' scales, specifically designed for a disease, in this case obesity. Although specific scales are seen to be well adapted to the disease they are designed for and better at recording even minor QoL changes, they are sometimes considered to be quite tautological, producing repetitive and difficult to interpret results. In contrast, generic scales may be poorly adapted to particular conditions of a disease and lack sensitivity. These issues not only bring about the debate of which scales are best to use when, but also bring into question whether together these two types of scales provide coherent information [68]. Moreover, there is a general lack of consensus on the definition of QoL despite its importance for evaluating quality and outcome of health which means there is no gold standard for its measurement [69-71].

A number of reasons have been attributed to persistent psychological issues post-surgery including unrealistic patient expectations [72]. These findings point to a need for greater modelling of the psychological pathway to obesity in order to potentially improve treatment outcomes. Work in this field is currently limited and unfortunately, there is no single set of psychological characteristics that are consistently predictive of success or failure following BS as 
Running Title: Bariatric Surgery and long term Psychosocial Quality of Life

different psychological characteristics are likely to be associated with weight maintenance and relapse in obesity [73]. This review points to potential for exploratory work with both patients and health professionals around psychological pathways in the use of longer term quality of life measures, the adoption of a life course view of obesity. The work also confirms the value of existing psychological work with this group when treatment costs require clear evidence of effectiveness. There may also be a role for enhanced psychological support to address postoperative mental health issues. More recently, studies have reported elevated suicide rates following bariatric surgery [74-75] in BS groups when compared to control groups [76].

\section{Limitations}

Several limitations to the review findings must be noted. Firstly, the limited number of RCTs may explain for the high risk of allocation bias of participants to intervention and control groups in the review studies, but also contribute to selection bias. Allocation of participants to groups presented the most common risk of bias to the review studies, as participants generally chose which group they could go into. For example in Karlsson et al [39], participants could volunteer for conventional or surgical treatment. Thereafter the type of surgery they had was up to the surgeon's preference. The retrospective collection of data in Batsis et al [53] meant participants had already previously undergone RYGBP or not when they were approached to participate. Buddeberg-Fischer [54] included patients applying for BS. However, comparability of the surgery and no-surgery groups was unclear because baseline characteristics for each group were not reported, although the study mentions that all but three participants met the criteria for surgery (one had BMl 31.6, two were aged > 60 years). There was a lack of evidence on the standardisation of the surgical interventions given i.e. whether one surgeon was conducting a surgical procedure similarly to another. This problem was also evident for the non-surgical interventions which included a mixture of unknown conventional treatments [39] and a weight- 
Running Title: Bariatric Surgery and long term Psychosocial Quality of Life

loss program [55]. Differences like these reduce true comparability between surgery and no surgery, increasing the risk of selection bias [77] potentially influencing skewed significant results, and affecting generalisability of findings. As for the RCTs a high percentage of potential participants refused to participate in the trials which contributed to small sample sizes therefore reported results should be considered preliminary [51-52].

Other overall problems of studies in this field include variation in sample sizes, follow up periods and study design. Such statistical and methodological heterogeneity limits the ability to provide quantitative synthesis of the results as caution has to be raised over comparability of the studies' results [78]. The risk of language bias is also quite prominent in this review as studies published in English only were included. Lastly this review aimed to look at long term psychosocial QoL following bariatric surgery, however, only five out of the nine studies included follow up data for more than three years. All the above issues potentially weaken the strength of evidence within this field therefore there is a need for more longitudinal prospective studies to be conducted in this area.

\section{Conclusion}

- From the small numbers of strong to moderate quality studies found in this review, no strong conclusions can be drawn regarding the effectiveness of BS compared to nonsurgical interventions on improving long term psychosocial QoL of obese adults due to limited data.

- More research needs to be conducted in this area to gain greater understanding of the effects BS has on psychosocial QoL using higher quality study designs like RCTs and controlled trials whilst accounting for ethical issues that randomisation could present, for example denying some participants clinically indicated surgical treatment. 
- There is a need to clarify the definition of (psychosocial) QoL to enable researchers to develop and establish a set of valid and reliable questionnaires in this area.

- Aside from time, additional patient attributes like race, gender, socioeconomic status and mental health status may influence psychosocial QoL outcomes post-surgery therefore greater specificity on the predictors of post-BS QOL may help produce clearer findings.

- In future it would be good to pull together data on trends in QoL verses weight loss following BS and map out specific time points were QoL peaks or significantly drops. This data would be useful for health professionals to map out appropriate timescales for psychological interventions.

\section{Disclosure Statement}

The authors declare no conflicts of interest. 


\section{REFERENCES}

1. The NHS Information Centre. Health Survey for England - 2008, physical activity and fitness; vol. 2 - methods and documentation.

https://catalogue.ic.nhs.uk/publications/public-health/surveys/heal-surv-phys-acti-fitneng-2008/heal-surv-phys-acti-fitn-eng-2008-rep-v3.pdf (accessed on 31.12.13).

2. Sutton R. Adult anthropometric measures, overweight and obesity. The Health and Social Care Information Centre; 2011. Retrieved from https://catalogue.ic.nhs.uk/publications/public-health/surveys/heal-surv-eng2011/HSE2011-Ch10-Adult-obesity.pdf

3. Ogden CL, Yanovski SZ, Carroll MD, Flegal KM. The epidemiology of obesity. Gastroenterology, 2007; 132(6):2087-102.

4. Salome CM, King GG \& Berend N. Physiology of obesity and effects on lung function. Journal of Applied Physiology, 2010; 108:206-11.

5. Whiteman DC, Sadeghi S, Pandeya N, Smithers BM, Gotley DC, Bain CJ,et al. Combined effects of obesity, acid reflux and smoking on the risk of adenocarcinomas of the oesophagus. Gut, 2008; 57(2):173-80

6. Griffiths LJ, Parsons TJ, Hill AJ. Self-esteem and quality of life in obese children and adolescents: a systematic review. International Journal of Pediatric Obesity, 2010; 5(4):282-304.

7. Wang F, Wild TC, Kipp W, Kuhle S, Veugelers PJ. The influence of childhood obesity on the development of self-esteem. Statistics Canada, Health Reports, 2009; 20(2):21-7. http://www.statcan.gc.ca/pub/82-003-x/82-003-x2009002-eng.pdf (Accessed on 27 Jan 2014)

8. Puhl RM \& Heuer CA. Obesity Stigma: Important Considerations for Public Health. American Journal of Public Health, 2010; 100(6):1019-1028. doi: 10.2105/AJPH.2009.159491. PMCID: PMC2866597 
9. Maggard MA, Shugarman LR, Suttorp M, et al. Meta-analysis: surgical treatment of obesity. Annals of Internal Medicine, 2005; 142(7):547-59.

10. Dombrowski SU, Knittle K, Avenell A, Araújo-Soares V \& Sniehotta FF. Long term maintenance of weight loss with non-surgical interventions in obese adults: systematic review and meta-analyses of randomised controlled trials. British Medical Journal, 2014; 348:g2646. doi: 10.1136/bmj.g2646.

11. NICE Guidelines (CG189) - Obesity: identification, assessment and management of overweight and obesity in children, young people and adults, Nov 2014 http://www.nice.org.uk/guidance/cg189/evidence/cg189-obesity-update-full-guideline2

12. Shaffer EA. Bariatric Surgery: A Promising Solution for Nonalcoholic Steatohepatitis in the Very Obese. Journal of Clinical Gastroenterology, 2006; 40: S44-S50. DOI: 10.1097/01.mcg.0000168649.79034.2e

13. HSCIC Finished Consultant Episodes for bariatric surgery 2002-03 to 2012-13 http://www.hscic.gov.uk/article/2021/WebsiteSearch?q=Finished+Consultant+Episodes+for+bariatric+surgery\&go=Go\&area=both

14. Statistics on Obesity, Physical Activity and Diet - England, 2015 [NS] Publication date: March 03, 2015 http://www.hscic.gov.uk/catalogue/PUB16988/obes-phys-acti-diet-eng-2015.pdf

15. Buchwald H \& Oien DM. Metabolic/Bariatric Surgery Worldwide 2011, Obesity Surgery, 2013;23(4): 427-436. DOI: 10.1007/s11695-012-0864-0

16. Robinson MK. Editorial: Surgical treatment of obesity - weighing the facts. New England Journal of Medicine 2009; 361(5):520-21. doi:10.1056/NEJMe0904837.PMID 19641209.

17. Buchwald $\mathrm{H}$, Avidor $\mathrm{Y}$, Braunwald $\mathrm{E}$, et al. Bariatric Surgery: A Systematic Review and Meta-analysis. JAMA. 2004; 292(14):1724-1737. doi:10.1001/jama.292.14.1724. 
18. Colquitt JL, Pickett K, Loveman E, Frampton GK. Surgery for weight loss in adults. Cochrane Database of Systematic Reviews 2014; Issue 8. Art. No.: CD003641. DOI: 10.1002/14651858.CD003641.pub4.

19. Picot J, Jones J, Colquitt JL, et al. The clinical effectiveness and cost-effectiveness of bariatric (weight loss) surgery for obesity: a systematic review and economic evaluation. Health Technology Assessment, 2009; 13(41):1-190, 215-357, iii-iv. doi: 10.3310/hta13410.

20. Engel GL. The need for a new medical model: a challenge for biomedicine. Science 1977; 196(4286): 129-36.

21. Nouri FM, Lincoln NB. An extended Activities of Daily Living scale for stroke patients. Clinical Rehabilitation 1987; 1: 301-05.

22. McDowell I, Newell c. Measuring Health: A guide to Rating Scales and Questionnaires. Oxford: Oxford University Press, 1987.

23. Bowling A. Measuring Health. A Review of Quality of Life Measurement Scales (2nd edition). Buckingham: Open University Press, 1997.

24. Roberts R, Towell T, Golding J. Foundations of Health Psychology. Houndsmill, Basingstoke: Palgrave, 2001.

25. Westcott H, Cross M. This Far and No Further: Towards Ending the Abuse of Disabled Children. Birmingham: Venture Press, 1996.

26. Muldoon MF, Barger SD, Flory JD, Manuck SB. What are quality of life measurements measuring? BMJ 1998; 316(7130): 542-45 
27. Whitehouse CR. The teaching of communication skills in United Kingdom medical schools. Med Educ 1991; 25(4): 311-18.

28. Llewelyn SP. Caring: the cost to nurses and relatives. In: Broome A, editor. Health Psychology: Processess and Applications. London: Chapman Hall, 1989.

29. Mitchell JE, King WC, Courcoulas A, et al. Eating behavior and eating disorders in adults before bariatric surgery. International Journal of Eating Disorder, 2015; 48:215-222. doi: 10.1002/eat.22275

30. Lier H Ø, Biringer E, Stubhaug B \& Tangen T. Prevalence of psychiatric disorders before and 1 year after bariatric surgery: The role of shame in maintenance of psychiatric disorders in patients undergoing bariatric surgery. Nordic Journal of Psychiatry, 2013;67:89-96.

31. Butland B, Jebb S, Kopelman P, et al. Tackling obesities: future choices - project report (2nd Ed). London: Foresight Programme of the Government Office for Science; 2007. Retrieved from www.bis.gov.uk/assets/bispartners/foresight/docs/obesity/17.pdf

32. Markowitz S, Friedman MA, Arent SM. Understanding the relation between obesity and depression: Causal mechanisms and implications for treatment. Clinical Psychology: Science and Practice 2008; 15(1):1-20.

33. Napolitano MA, Foster GD. Depression and obesity: Implications for assessment, treatment, and research. Clinical Psychology: Science and Practice 2008;15(1):21-27.

34. Incledon E, Wake M, Hay M. Psychological predictors of adiposity: systematic review of longitudinal studies. International Journal of Pediatric Obesity. 2011 Jun;6(2-2):e1-11. doi: 10.3109/17477166.2010.549491. Epub 2011 Jan 19. 
Running Title: Bariatric Surgery and long term Psychosocial Quality of Life

35. Collins JC, Bentz JE. Behavioral and Psychological Factors in Obesity. The Journal of Lancaster General Hospital, 2009; 4(4):124-7.

36. Byrne SM, Cooper Z \& Fairburn CG. Psychological Predictors of Weight Regain in Obesity. Behaviour Research and Therapy, 2004; 42(11):1341-56.

37. National Institute for Health and Care Excellence. Bariatric surgical service for the treatment of people with severe obesity - Commissioning Guidance, London: National Institute for Health and Care Excellence; 2007.

38. NHS Choices. Weight loss surgery - Life after surgery. http://www.nhs.uk/Conditions/weight-loss-surgery/Pages/Recommendations.aspx. (Accessed 12 Feb 2014)

39. Karlsson J, Taft C, Ryden A, Sjostrom L, Sullivan M. Ten-year trends in health-related quality of life after surgical and conventional treatment for severe obesity: the SOS Intervention study. International Journal of Obesity, 2007; 31:1248-61.

40. Heddens CJ \& Vonnahme C. A Journey Through Massive Weight Loss, Bariatric Nursing and Surgical Patient Care, 2006; 1.1: 31-38

41. Birch A, Ogden J, Wood K. 'The wrong journey': patients' experience of plastic surgery post weight loss surgery. Qualitative Research in Sport, Exercise and Health. 2015; 7(2)

42. Andersen Andersen JR, Aasprang A, Karlsen T, et al Health-related quality of life after bariatric surgery: a systematic review of prospective long-term studies. Surgery for Obesity and Related Diseases, 2015; 11(2): 466-473

43. Hollywood A, Ogden J, Pring C. The impact of a bariatric rehabilitation service on weight loss and psychological adjustment - study protocol. BMC Public Health, 2012; 12(275). doi:10.1186/1471-2458-12-275 
44. Padwal RS, Majumdar SR, Klarenbach S, et al. The Alberta population-based prospective evaluation of the quality of life outcomes and economic impact of bariatric surgery (APPLES) study: background, design and rationale. BMC Health Services Research, 2004; 10:284. doi: 10.1186/1472-6963-10-284

45. Thomas BH, Ciliska D, Dobbins M, Micucci S. A process for systematically reviewing the literature: Providing the research evidence for public health nursing interventions. Worldviews on Evidence-Based Nursing, 2004; 1(3):176-84.

46. Armijo-Olivo S, Stiles CR, Hagen NA, Biondo PD, Cummings GG. Assessment of study quality for systematic reviews: a comparison of the Cochrane Collaboration Risk of Bias Tool and the Effective Public Health Practice Project Quality Assessment Tool: methodological research. J Eval Clin Pract. 2012; 18(1): 12-18. doi: 10.1111/j.13652753.2010.01516.x

47. CRD's guidance for undertaking reviews in health care. Accessed online at: http://www.york.ac.uk/crd/SysRev/!SSL!/WebHelp/SysRev3.htm

48. Haynes RB, Ackloo E, Sahota N, McDonald HP, Yao X. Interventions for enhancing medication adherence. Cochrane Database of Systematic Reviews 2008, Issue 2. doi/10.1002/14651858.CD000011.

49. Kontopantelis E, Springate DA, Reeves D. A Re-Analysis of the Cochrane Library Data: The Dangers of Unobserved Heterogeneity in Meta- Analyses. PLoS ONE. 2013; 8(7): e69930. doi:10.1371/journal.pone.0069930 
50. Petticrew M \& Roberts H. Synthesizing the Evidence, in Systematic Reviews in the Social Sciences: A Practical Guide. Blackwell Publishing Ltd, Oxford, UK. 2006. doi: 10.1002/9780470754887.ch6

51. Ding SA, Simonson DC, Wewalka M, et al. Adjustable Gastric Band Surgery or Medical Management in Patients With Type 2 Diabetes: A Randomized Clinical Trial. J Clin Endocrinol Metab. 2015;100(7):2546-2556. doi: 10.1210/jc.2015-1443. Epub 2015 Apr 24.

52. Halperin F, Ding S, Simonson et al. Roux-en-Y Gastric Bypass Surgery or Lifestyle With Intensive Medical Management in Patients With Type 2 Diabetes: Feasibility and 1-Year Results of a Randomized Clinical Trial. JAMA Surg. 2014; 149(7): 716-726.

doi: 10.1001/jamasurg.2014.514

53. Batsis JA, Lopez-Jimenez F, Collazo-Clavell ML, Clark MM, Somers VK, Sarr MG. Quality of life after bariatric surgery: a population-based cohort study. American Journal of Medicine, 2009; 122(11):1055.e1-1055.e10. doi: 10.1016/j.amjmed.2009.05.024.

54. Buddeberg-Fischer B, Klaghofer R, Krug L, et al. Physical and psychosocial outcome in morbidly obese patients with and without bariatric surgery: a 4 1/2-year follow-up. Obesity Surgery, 2006; 16(3):321-30.

55. Canetti L, Berry EM, Elizur Y. Psychosocial predictors of weight loss and psychological adjustment following bariatric surgery and a weight-loss program: the mediating role of emotional eating. International Journal of Eating Disorders, 2009; 42(2):109-17. doi: 10.1002/eat.20592.

56. Nickel C, Widermann C, Harms D, et al. Patients with extreme obesity: change in mental symptoms three years after gastric banding. International Journal of Psychiatry Medicine, 2005; 35(2):109-22.

57. Kolotkin RL, Crosby RD, Gress RE, Hunt SC \& Adams TD. Two-year changes in healthrelated quality of life in gastric bypass patients compared with severely obese controls. Surgery for Obesity and Related Diseases, 2009; 5(2):250-56. doi:

10.1016/j.soard.2009.01.009. 
58. Adams TD, Pendleton RC, Strong MB, et al. Health outcomes of gastric bypass patients compared to nonsurgical, non-intervened severely obese. Obesity (Silver Spring), 2010; 18(1):121-30. doi: 10.1038/oby.2009.178.

59. Adams TD, Davidson LE, Litwin SE, et al. Health benefits of gastric bypass surgery after 6 years. JAMA, 2012; 308(11):1122-31.

60. Kolotkin RL, Davidson LE, Crosby RD, Hunt SC, Adams TD. Six-year changes in healthrelated quality of life in gastric bypass patients versus obese comparison groups. Surgery for Obesity and Related Diseases 2012; 8(5):625-33. doi: 10.1016/j.soard.2012.01.011.

61. Hell E, Miller KA, Moorehead MK \& Samuels N. Evaluation of Health Status and Quality of Life after Bariatric Surgery: Comparison of Standard Roux-en-Y Gastric Bypass, Vertical Banded Gastroplasty and Laparoscopic Adjustable Silicone Gastric Banding. Obesity Surgery, 2000; 10(3): 214-219

62. Romy S, Donadini A, Giusti V \& Suter M. Roux-en-Y Gastric Bypass vs Gastric Banding for Morbid Obesity A Case-Matched Study of 442 Patients, Arch Surg. 2012; 147(5):460-466. doi:10.1001/archsurg.2011.1708.

63. Kalfarentzos F, Skroubis G, Kehagias I, Mead N \& Vagenas K. A prospective comparison of vertical banded gastroplasty and Roux-en-Y gastric bypass in a non-superobese population. Obesity Surgery, 2006; 16(2):151-158

64. Nguyen NT, Slone JA, Nguyen XT et al. A Prospective Randomized Trial of Laparoscopic Gastric Bypass Versus Laparoscopic Adjustable Gastric Banding for the Treatment of Morbid Obesity: Outcomes, Quality of Life, and Costs. Annals of Surgery, 2009; 250(4): 631-641. doi: 10.1097/SLA.0b013e3181b92480

65. Bowne WB, Julliard K, Castro AE et al. Laparoscopic gastric bypass is superior to adjustable gastric band in super morbidly obese patients: A prospective, comparative analysis. Arch Surg. 2006; 141(7):683-9 
66. Glinski J, Wetzler S, Goodman E. The psychology of gastric bypass surgery. Obesity Surgery, 2001; 11:581-588.

67. Sarwer DB, Fabricatore AN, Jones-Corneille LR, Allison KC, Faulconbridge LN, Wadden TA. Psychological Issues Following Bariatric Surgery. Primary Psychiatry, 2008; 15(8):50-5.

68. Le Pen C, Levy E, Loss F, Banzet MN \& Basdevant A. "Specific" scale compared with "generic" scale: a double measurement of the quality of life in a French community sample of obese subjects. Journal of Epidemiology Community Health, 1998; 52(7): 445450. PMCID: PMC1756735

69. Schalock, RL. The Concept of Quality of Life: What We Know and Do Not Know. Journal of Intellectual Disability Research, 2004; 48(3):203-16

70. Moons P, Budts W, De Geest S. Critique on the conceptualisation of quality of life: a review and evaluation of different conceptual approaches. International Journal of Nursing Studies, 2006; 43(7):891-901

71. Simon SD. Is the randomized clinical trial the gold standard of research? Journal of Andrology, 2001; 22(6): 938-43

72. Kubik JF, Gill RS, Laffin M, Karmali S. The Impact of Bariatric Surgery on Psychological Health. Journal of Obesity, 2013; doi: 10.1155/2013/837989

73. Van Hout GCM, Verschure SK, van Heck GL. Psychological predictors of success following bariatric surgery. Obesity Surgery, 2005; 15(4): 552-60.

74. Tindle HA, Omalu B, Courcoulas A, et al. Risk of suicide after long-term follow-up from bariatric surgery. The American Journal of Medicine. 2010;123(11):1036-42. doi: 10.1016/j.amjmed.2010.06.016.

75. Peterhansel C, Petroff D, Klinitzke G, Kersting A, Wagner B. Risk of completed suicide after bariatric surgery: a systematic review. Obesity Reviews 2013; 14(5): 369-382 
76. Adams TD, Gress RE, Smith SC, et al. Long-Term Mortality after Gastric Bypass Surgery. The New England Journal of Medicine2007; 357 : 753-761. DOI: 10.1056/NEJMoa066603

77. Bailey L, Vardulaki K, Langham J, Chandramohan D. Introduction to Epidemiology, Open University Press in collaboration with LSHTM, London; 2005.

78. Impellizzeri FM, Bizzini M. Systematic review and meta-analysis: a primer. International Journal of Sports Physical Therapy, 2012; 7(5):493-503.

Shugarman LR, Suttorp M, Maglione M, Sugerman HJ, Livingston EH, Nguyen NT, Li Z, Mojica WA, Hilton L, Rhodes S, Morton SC, Shekelle PG. 